\title{
Relational Matching-to-sample Concept Learning in Children
}

\author{
Md. Abu Bokor Siddik1,*, Habiba Aktar Lata1, Abu Yusuf Mahmud² \\ ${ }^{1}$ Department of Psychology, Rajshahi College, Bangladesh \\ ${ }^{2}$ Department of Psychology, University of Dhaka, Bangladesh
}

Copyright $\bigcirc 2019$ by authors, all rights reserved. Authors agree that this article remains permanently open access under the terms of the Creative Commons Attribution License 4.0 International License

\begin{abstract}
The present study was formulated with an aim to examine the identical relational matching-to-sample concept learning in children, in which five students of kindergarten aged 7-8 were concurrently trained with two-choice relational matching-to-sample (RMTS) tasks by using various shapes as stimuli under the conditions of no reinforcement for making correct responses. In particular, the RMTS tasks allowed subjects to see a sample of pair of stimuli at first, and then two comparison pair of stimuli. Subjects were trained to choose one of the two comparison pair of stimuli that matched the sample one. The number of tasks (e.g., AA vs. BB \& MA in phase 1 ; BB vs. AA \& MA in phase 2) was gradually increased. Research findings showed around or more than chance level performances made by three subjects out of five during the initial performances of the acquisition training in phase 1(e.g., AA vs. BB \& MA). Subsequently, subjects were able to rapidly master the acquisition tasks with higher accuracy. After learning these RMTS tasks, they transferred the discriminative responding to two novel sets of stimuli securing higher accuracy in both baseline and test performances suggesting that they might have perceived and compared the relationship between objects in the sample pair of stimuli to the relationship between objects in the comparison pair of stimuli. Our findings suggest that children seemed to adopt the relational strategy to learn the RMTS tasks. These findings are considered in light of the implications for understanding the nature of the relational learning and their evolutionary origins.
\end{abstract}

Keywords Same/ Different Concept, Relational Matching-to-sample, Identity Matching-to-sample, Concurrent Presentation, Stimulus Generalization

\section{Introduction}

Abstract concept learning, rules about relationship (e.g., identical, different) among stimuli, has been a central focus in comparative cognition studies because it demonstrates to novel stimuli that transcends concrete experiment (Katz, Wright \& Bodily [1]). One of the most popular and powerful means of studying relational concepts is the Same/Different concept (S/D) that shows an ability to identify S/D stimulus from item pairs and its successful transfer to novel stimuli. There are several procedures of studying S/D concepts (e.g., S/D discrimination, Matching-to-Sample, oddity discrimination). As a measure of conditional discriminations and concept learning in humans and nonhuman animals, matching-to-sample (MTS) tasks have been using over a half century (Wright \& Lickteig [2]). The MTS procedure is a kind of conditional learning as it provides an advantage to be produced conditional relations between or among stimuli. In detail, a sample stimulus (e.g., a cup) is followed by two comparison stimuli (e.g., a cup and a spoon) in MTS tasks. Choosing one (e.g., a cup) of the two comparison stimuli that resembles the sample one is considered correct response.

It has been studied in a variety of ways such as identity matching-to-sample (IMTS, in which responding to one of the two comparison stimuli matching the sample is the correct answer), oddity from sample (OFS, in which responding to one of the two comparison stimuli mismatching from the sample is the correct answer), arbitrary matching-to-sample (AMTS, in which subjects are reinforced for arbitrarily choosing one [e.g., a black cross] of the two comparison stimuli [e.g., a black cross and a black square] in the presence of a sample stimuli [e.g., a red circle]), and relational matching-to-sample (RMTS) to gain an understanding of comparative learning abilities of animals (e.g., Wright \& Lickteig, [2] with pigeons; D'Amato, Salmon, \& Colombo [3] with monkeys; Kastak, \& Schusterman [4] with California sea lion). As compared to animals, several attempts were made to investigate MTS learning abilities in humans (e.g., Boelens \& Schenk [5]; Lazar [6]; Weinstein [7] with rhesus and children).

But the experimental procedures particularly in the case 
of IMTS the previous studies (e.g., Wright \& Lickteig [2]; D'Amato et al. [3]; Kastak, Schusterman [4]) employed seemed to be implicated with some perceptual cues that might pave the way the experimental subjects to solve the MTS tasks thus contributing to the concept formation influenced by perceptual modality (specific physical features of the stimuli). In detail, matching to sample procedures may produce short-term familiarity prevailing among the sample and comparison stimuli that might be in operation in experimental subjects as discriminative cues for solving the MTS tasks (Premack, [8]). In MTS method, sample is presented twice: Once as a sample stimulus and the next time as one of the two comparison stimuli (see figure 1).

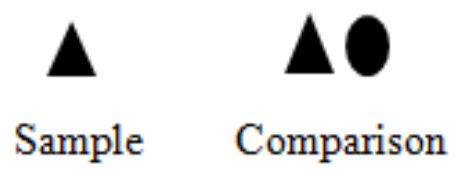

Figure 1. An Example of MTS Tasks

With such advantage, subjects may feel different level of familiarity between the sample and the matching stimuli. Against such backdrop, relational matching-to-sample (RMTS) tasks, first invented by Premack [8] to explore the representation same and different in nonhuman primates, might be considered. In RMTS tasks, at first, a pair of sample stimulus (AA) appeared and then two pair of comparison stimuli (BB and $\mathrm{CD}$ ) appeared. Subjects require choosing one $(\mathrm{BB})$ of the two comparison stimuli (BB \& CD) that matches the sample (AA). On the contrary, if the sample is $\mathrm{AB}$, the correct choice is $\mathrm{CD}$ rather than BB.As compared to MTS and non MTS (to choose one of the two comparison stimuli that is different from the sample), summary mental symbols of the representation of same and different is in operation in RMTS tasks. In addition, RMTS tasks follow the strategy of a structure mapping analogy in which a correct choice requires building an understanding between two items in the array rather than responding to the items based on the stimuli features making it an array of analogical reasoning (Premack [8]). The RMTS has the potentiality to test an early relational ability. More particularly, it has an ability to explore arguably the simplest and the most basic relation. Furthermore, it is easily perceptually available to infants and young children (Ferry, Hespos \& Gentner, unpublished data; Smith [9]) and to other species (Fagot \& Thompson [10]; Wright \& Katz [11]). It gives birth another merit, that is, no specific experimental knowledge such as causal knowledge is needed to study the identity relation. There is no room for competing similiarity choices in RMTS tasks.

A number of researches (e.g., Martinho \& Kacelnik [12]; Thompson, oden, \& Boysen [13]) were carried on with these procedures. Recently, Martinho and Kacelnik [12] employed relational matching-to-sample (RMTS) method, a second-order relationship, to examine same/different concept in a new variety of species called ducklings (new born) without reinforced training. They presented a same pair of stimuli (e.g., two red ovals) to new born ducklings as a sample and then two comparison stimuli, one out of which was two blue ovals and another one was a different pair of stimuli (e.g., a triangle shape and a rectangle shape). In another trial, they presented a different pair of stimuli (e.g., (6) as sample one and then did two comparison stimuli (e.g., 6 vs. which matched the sample one. A response to one of the two comparison stimuli that matched the sample one was considered a correct response.

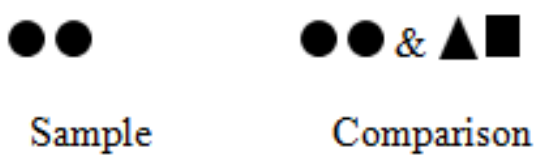

Figure 2. An example of RMTS employed by Martinho and Kacelnik [12]

It was observed in Martinho and Kacelnik's study [12] that the perceptual features of sample stimuli ( ) and the matching-to-sample stimuli $(\mathbf{O} \& \Delta)$ shared some common features (e.g., both shapes were ovals). Hence, it is acknowledged that although the color of the oval stimuli (sample) is different from other oval stimuli (as a part of two comparison stimuli), a particular feature of the stimuli (oval shape) might guide ducklings' discriminative behavior towards attaining S/D concept learning. Obozova, Smirnova, Zorina and Wasserman's study [14] who applied such RMTS protocol to two juvenile orange-winged amazons showed reliable performances to matching-to-sample tasks. They designed the presentation of stimuli in such a way that kept little room for sharing any common features between sample and matching-to-sample stimuli or sample and oddity from sample stimuli particularly in relational trials (e.g., a pair of red circles as sample stimuli and a pair of blue squares along with a pair of different shapes namely a rectangle and a triangle as comparison stimuli) that considerably resembled to the research design of the present study particularly in case of identity RMTS (choosing one of the two comparison stimuli that matches the sample). Relational matching procedure has been tested across different species (e.g., crows, apes, ducklings, amazons).

Like animals, a handsome number of RMTS studies with humans were administered (e.g., Christie \& Gentner [15]; Hochmann, Tuerk, Sanborn, Zhu, Long, Dempster \& Carey [16]; Walker, Bridgers \& Gopnik [17]; Walker \& Gopnik [18]). Recently, Christie and Gentner [15] administered five experiments on identical RMTS in children aged 2-4. They, at first, (as a part of experiment 1) presented standard and then two alternatives (one was relational matches $[R$ in which selecting one of the two alternatives matching the standard was the correct answer] and another one was non-relational [NR in which an item mismatched the standard]). Subjects were instructed to make their choices by pointing to one of the two 
alternatives. They did not receive any feedback for their responding. The first stage of Experiment 2 involved four corrective feedback trials that allowed 2-year olds and 3 -year olds to make an understanding regarding a correct choice. The second stage involved eight relational matching trials plus two catch trials as in Experiment 1 with no corrective feedback. Experiment 3 was identical to Experiment 1. One key feature of this experiment included naming session in which subjects were asked to utter the word "Same" when they saw two objects (e.g., two identical balls) containing same appearances. Afterwards, they were shown a sample different card (e.g., a red brush and a red bottle) and made them utter the word "Different". Experiment 4 was identical to Experiment 1 except that the former showed a standard and made the subjects utter the word used as the standard. Following this, two choices (one matched the standard and another one did not) appeared.

However, with these experiments, Christie and Gentner's studies [15] revealed that subjects under four years of age could not match the standard with one of the two alternatives that matched the standard without feedback (although initial feedback was provided in Experiment 2 and Experiment 3). But when children aged 5-6 acquired symbolic -linguistic experiences, they were able to process the RMTS tasks relationally. More recently, to explore the preschoolers' abilities to solve the array match-to-sample (AMTS) problems, Hochmann, Tuerk, Sanborn, Zhu, Long, Dempster \& Carey [16] carried on five experiments in which as a part of Experiment 1 16-item same and 16-item different arrays of stimuli were employed. Comparison stimuli were followed by sample stimuli with feedback at training phase. Test trials were similar to the training trials except no feedback was given. In Experiment 2, sample stimuli (2-item array same card) were followed by two comparison stimuli (2-item comparison stimuli) with verbal reinforcement. Testing phase was identical to training phase except no feedback was delivered. Experiment 3 was similar to that of Experiment 1 except the former had eight training trials (four 16-same sample cards and four 16-different sample cards) with feedback and had 20 test trails. Experiment 4 was identical to Experiment 2 and Experiment 3 with an exception that it tested 3-4-5 year olds on 2-item AMTS after training with on 16-item AMTS. Experiment 5 examined 3 and 4 year olds' performances on all the same vs. 13 same/3 different AMTS tasks and subsequently, on 2-item AMTS task. The overall findings of these experiments demonstrated that robust success (good transfer of learning to the test stimuli) in justifying the discrimination tasks based on the relationship of same and different was not observed in 3-4 year olds but these relational abilities were clearly visible in adults.

It is noticeable that most of the RMTS studies have been tested with humans belonging to the western cultures. There is hardly any RMTS studies tested in light of the cognitive development of children living in non western cultures particularly in south Asian cultures. A number of behavioral studies (Chiao \& Blizinsky [19]) and cognitive studies (Chua, Boland \& Nisbett [20]; Masuda \& Nisbett $[21,22])$ showed cross-cultural differences in adults. Kuwabara and Smith's study [23] demonstrated differences in attentional and cognitive processing of adults from Eastern and Western cultures. More specifically, their studies found the cognition of Eastern adults more relational than that of Western adults. Thus the present study aims to test the RMTS of Bangladeshi children. In particular, the present study seeks to examine the cognitive development and the higher-order cognitive abilities of Bangladeshi children. Through such research works, this study would present a little view on how much higher-order learning abilities Bangladeshi children have or deepening our understanding about the cognitive development of Bangladeshi children as compared to western countries. In addition, it will strengthen Bangladesh government's effort towards children in terms of the development of health, education and so forth .The development origins and timing of these differences plays a significant role in understanding the role of culture in cognition and to understanding the developmental processes in greater extent. Relational studies have significant importance on an understanding of cognitive developmental difficulties in children. It is reported (Smith \& Tunick [24]) that retarded children have tendency to acquire the relational tasks based on specific cues of the stimuli rather than the relationship prevailing among the stimuli.

We hypothesize that the children used in the present study are expected to learn RMTS tasks because Bangladeshi children of some urban areas are now getting the education materials (e.g., books, syllabus, lecture style) and congenial environment for education that are somewhat similar to that of western countries by dint of globalization.

The present study employed AA $(O)$ as a pair of sample stimuli and then BB ( $\square$ ) vs. MA ( $)$ as comparison stimuli.
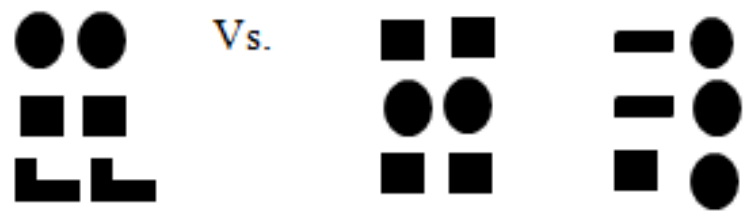

\section{Sample}

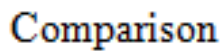

Figure 3. An example of stimuli presentation of the present study conducted by Siddik, Lata \& Mahmud

A response to one of the two comparison stimuli that relationally matched the sample one was considered a correct response. Such setting of stimuli hardly provides an 
opportunity to share some common features between sample and matching-to-sample stimuli thus eliminating the possibility of the perceptual effects on humans' concept formation. Although several studies were able to demonstrate S/D concept learning (MTS/RMTS) in human children, a major challenge is to confirm whether such concept formation by human children is conceptual or perceptual. The attainment of these challenges may properly assess this critical issue in human cognition. Stimuli were intermixed in the different pair of stimuli. For example, AA vs. BB \& MA was used in phase 1 task. Here, item A was used in both as sample and one of two comparison stimuli (MA). We used the same item (A) concurrently (an item that is positive in a trial becomes negative in another trial) to avoid the possibility of the specific features of a stimulus becoming discriminative cues. Furthermore, this provision might generate lesser amount of variation/novelty in the stimuli set that might make a little opportunity for perceptual cues to influence over the subjects' discriminative behavior. The more intensity (e.g., color, shape) a stimulus contains, the more attention will be paid by the subjects to that stimuli that (attention) might act as an effective discriminative cue to choose the correct stimulus. It was claimed (Birkimer [25]) that subjects' better responses to the discriminative stimuli based on what element was of the highest intensity thus resulting in much attention to the stimuli and causing better performances.

Another noticeable feature of the present study was that it did not have any provision of reinforcement in case of subjects making correct responses. Because we wanted to confirm that concept formation by human children could not be influenced by any factor. It is assumed that concept learning with reinforcement training did not seem to indicate the subjects' innate qualities rather it seemed to be the effect of the reinforcement. A learning experiment with reinforcement training allows the subjects to receive reinforcement for making correct responses at every trial. This advantage (receiving reinforcement) may give birth a sense among the subjects that may encourage them to make an association between correct stimuli and reinforcement rather than developing an understanding of relationship among stimuli. To avoid such possibility, we decided to conduct the experiment without reinforcement training.

Besides, we wanted to provide them a suitable atmosphere that can extract their natural qualities. We expected that human subjects would acquire this higher-order skill by utilizing their own efforts suggesting that they had this skill. Recently, Martinho and Kacelnik [12] reported relational matching-to-sample learning in newborn ducklings without reinforcement training suggesting that reinforcement could not be a determinant of acquiring relational concept. We investigated the same relationship among the RMTS tasks. Because abstract concept of sameness has prominently contributed to human development (e.g., Daehler \& Bukatko [26]). In addition, children develop cognition in stages and extend their abstract concept of sameness through using number, area and volume (Piaget \& Inhelder [27]). Regarding humans' cognition, William James [28] stated "The concept of sameness is very keel and backbone of our thinking".

\section{Method}

\subsection{Subjects}

Five children aged between 7 and 8 were used as subjects. They had freedom to pass all their time at home or school as per their choices except the duration of the experiment. An option to remain in the experiment until it reaches a desired goal or to give it up were kept open to all subjects. They had no previous experimental experience nor had they taken any courses in psychology. The proposed research selected the subjects with ages ranging from 7-8 because learning to form concept starts to emerge from the ages of 2 to 7 (Piaget [29]).

\subsection{Apparatus}

RMTS tasks were constructed from stimuli consisted of 13 shapes including some geometric ones (e.g., $\mathbf{O}$,

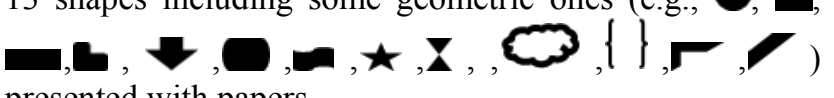

presented with papers.

\subsection{Procedures}

Students of kindergarten aged 7-8 were trained with two-choice matching-to-sample tasks using various shapes as stimuli. The experimenter, at first, presented a pair of sample stimuli and then did two pair of comparison stimuli (one same and one different). Choosing one of the two comparison stimuli that showed the same relationship as the sample one was considered correct response that was unreinforced. An incorrect response made by the subjects generated no punishment-like consequences. Training tasks started with phase 1 task in which, at first, AA (O) was presented as a pair of sample stimuli and then BB ( $\square$ ) vs. MA ( ) were presented as comparison stimuli. Subjects were trained in a daily session of 24 trials in total and participated in the discriminative tasks five to six times during the morning per week. A one minute inter-trial interval separated each trial. Tasks were increased gradually (e.g., $\mathrm{AA} / \mathrm{BB} / \mathrm{CC}$ vs. $\mathrm{CC} / \mathrm{AA} / \mathrm{BB} \&$ $\mathrm{MB} / \mathrm{MA} / \mathrm{AB}$ ). All pairs of stimuli (same pair of stimuli as sample one) in the tasks of the specific phase (e.g., phase 1 or phase 2) were equally presented. However, the learning criterion of phase 1 was $100 \%$ correct responses in a sessions that allowed subjects to be promoted to phase 2 in which the phase 1 tasks were given concurrently meaning that BB ( as a pair of sample stimuli and $\mathrm{AA}(\mathrm{C}$ )vs. MA( $)$ as comparison stimuli. In another trial, phase 1 task continued (AA vs. BB \& MA). One purpose of 
presenting the pair of stimuli concurrently was to avoid the possibility of a specific feature of the stimuli being used as an effective discriminative cue. When subjects were able to make $80 \%$ correct responses (learning criteria depends on the complexity of the learning tasks) in phase 2 tasks, phase 3 started where novel pair of stimuli CC ( 5 ) were added to the phase 1 and phase 2 tasks. More specifically, $\mathrm{CC}$ vs. AA \& MB, AA vs. CC \& $\mathrm{MA}$ and $\mathrm{BB}$ vs. AA \& AB were employed eight times each in a session. A successful completion of phase 3 tasks $(80 \%$ correct responses in a session) facilitated the subjects to be promoted to the tasks of phase 4 where novel pair of stimuli DD ( 7 ) were added to phase 1 , phase 2 , phase 3 tasks. Here, DD vs. AA \& MA, CC vs. BB \& AB, AA vs. DD \& $\mathrm{MB}$ and $\mathrm{BB}$ vs. $\mathrm{CC}$ $\& \mathrm{BD}$ were presented four times each in a session. On completion of learning criterion $(80 \%$ correct responses in a session) in the tasks of phase 4, transfer test 1 (intra-modal transfer) started where each novel (a task is made of stimuli that have never been seen before) task (e.g., EE $\star \star$ and FF $\mathbf{X}$ vs. UV $\square$ ) appeared twice after every six training tasks consisted of 24 RMTS tasks did in a session. For example, one novel task (e.g., EE $\star$ $\star$ and FF $\mathbf{X} \mathbf{X}$ vs. UV $\square$ ) six training tasks-one novel task six, (GG הח - six training tasks. In such manner, four novel tasks were inserted to the training tasks daily resulting in 28 trials (24 training tasks and 4 testing tasks comprising two sets of novel stimuli) in a session. It continued for ten days. Research procedures at a glance:

Phase 1. AA vs BB \& MA. Learning criteria (LC) was $100 \%$ correct responses.

Phase 2. BB vs AA \& MA (12 trials randomly) and AA vs. BB \& MA (12 trials randomly). LC was $80 \%$ correct responses.

Phase 3. CC vs. AA \& MB (eight trials randomly) or AA vs. $\mathrm{CC} \& \mathrm{MA}$ (eight trials randomly) or $\mathrm{BB}$ vs $\mathrm{AA} \& \mathrm{AB}$ (eight trials randomly). LC was $80 \%$ correct responses.

Phase 4. DD vs. AA \& MA (six trials randomly) or CC vs. $\mathrm{BB} \& \mathrm{AB}$ (six trials randomly) or AA vs. DD \& MB (six trials randomly) or $\mathrm{BB}$ vs. $\mathrm{CC} \& \mathrm{BD}$ (six trials randomly). LC was $80 \%$ correct responses.

Transfer test consisted of two novel tasks (EE vs. FF \& UV and GG vs. HH \& WX): Each novel task (e.g., EE vs. FF \& UV) was inserted twice after every six training tasks consisted of Phase 1, Phase 2, Phase 3 and Phase 4 tasks. It had been continuing for ten days.

\section{Results}

\subsection{Acquisition}

The initial (e.g., the first two sessions of phase 1) acquisition performances of the most of the subjects (S2, $\mathrm{S} 3$, and S4) revealed around or more than $50 \%$ correct choices (chance level) to the relational MTS tasks. All the subjects except S5 mastered phase 1 tasks with learning criteria (100\% correct responses) within three or four sessions. Interestingly, subsequent acquisition tasks (e.g., phase 2 , phase 3 , and phase 4 ) were rapidly learned by them with high accuracy $(100 \%$ correct $)$. As compared to others (S1, S3, and S4), S2's performances were little bit better. For example, he required one session to master each phase task (phase 2, phase 3, and phase 4). Conversely, the remaining three subjects ( $\mathrm{S} 1, \mathrm{~S} 3$, and $\mathrm{S} 4)$ took two or three sessions to learn phase 2 tasks. Afterwards, the rate of their (S1, S3 and S4) choice accuracy to training tasks improved. Consequently, they (S1, S3 and S4) acquired phase 3 and phase 4 tasks with one session. At a glance, their overall training performances indicated robust acquisition. The performances of S5 were not taken into consideration for further analysis as he voluntarily left the experiment.

\subsection{Transfer}

In transfer test, subjects were presented two sets of novel stimuli (EE vs. FF \& UV; GG vs. HH \& WX) along with the training tasks comprising 24 tasks. From the beginning of the transfer test, subjects made $100 \%$ choice accuracy to the novel sets of stimuli thus exhibiting robust transfer of learning. The transfer performances were consistent with the baseline performances (performances with training stimuli) thus revealing the acquisition of full concept learning.

\section{Discussion}

The present study presumably provided the first evidences of RMTS learning ability in children living in a south Asian country particularly in Bangladesh. The robust acquisition and transfer performances represent the significant transfer of learning (one tailed binomial test; ps<.05) suggesting that they not only learned to relationally process the acquisition tasks but also spread this relational strategy over novel stimuli set that confirmed RMTS ability in children. This document of RMTS was particularly noteworthy because our children could exhibit higher-order cognitive ability without reinforcement training that was also rare in human studies. Here it is noteworthy of mentioning that a handsome number of RMTS studies (Walker \& Gopnik [18]; Hochmann et al. [16]) have been conducted. Almost all of them provided feedback (either it was verbal or music) for making correct responses or to make them correct in responding to the discriminative stimuli. Among them, Christie and Gentner [15] conducted 4 experiments to examine RMTS learning abilities without feedback. But initial feedback was provided in Experiment 2 and Experiment 3.

At the initial stage of the RMTS tasks of the present study particularly in phase 1 , their responding strategies to phase 1 tasks were somewhat random indicating that they might have solved the tasks based on assumptions. In 
phase 2, they acquired the tasks within very few sessions (e.g., two sessions or three sessions). The acquisition of learning criteria in phase 1 was a remarkable point for the development of their performances, after which, they did not have to face any difficulty to master the RMTS tasks in phase 2, phase 3 and phase 4 and even in transfer test where stimuli sets were completely new to them (see figure 4 and figure 5). Hence, a question arises on why they faced difficulty to solve phase 1 tasks despite its becoming simple discrimination tasks consisted of two types of RMTS tasks (AA vs. BB \& MA; AA vs. MA \& $\mathrm{BB}$ ) as compared to phase 2 , phase 3 , and phase 4 tasks. One way to answer to the question may be that they might have developed abilities to identify and retain logical relations between pair of sample and comparison stimuli in phase 1, the reflection of which was observed in subsequent training tasks and in the successful transfer of learning comprising novel stimuli. In particular, their quick acquisitions in training tasks and successful transfer of learning to the novel stimuli sets revealed that children seemed to encode the relational properties from the training pair of stimuli and to generalize these experiences to sets of novel stimuli. The transfer of learning made by the children could be considered the full concept learning as the baseline performances (training performances during the testing phase) and transfer performances were equal and more than $80 \%$ correct.

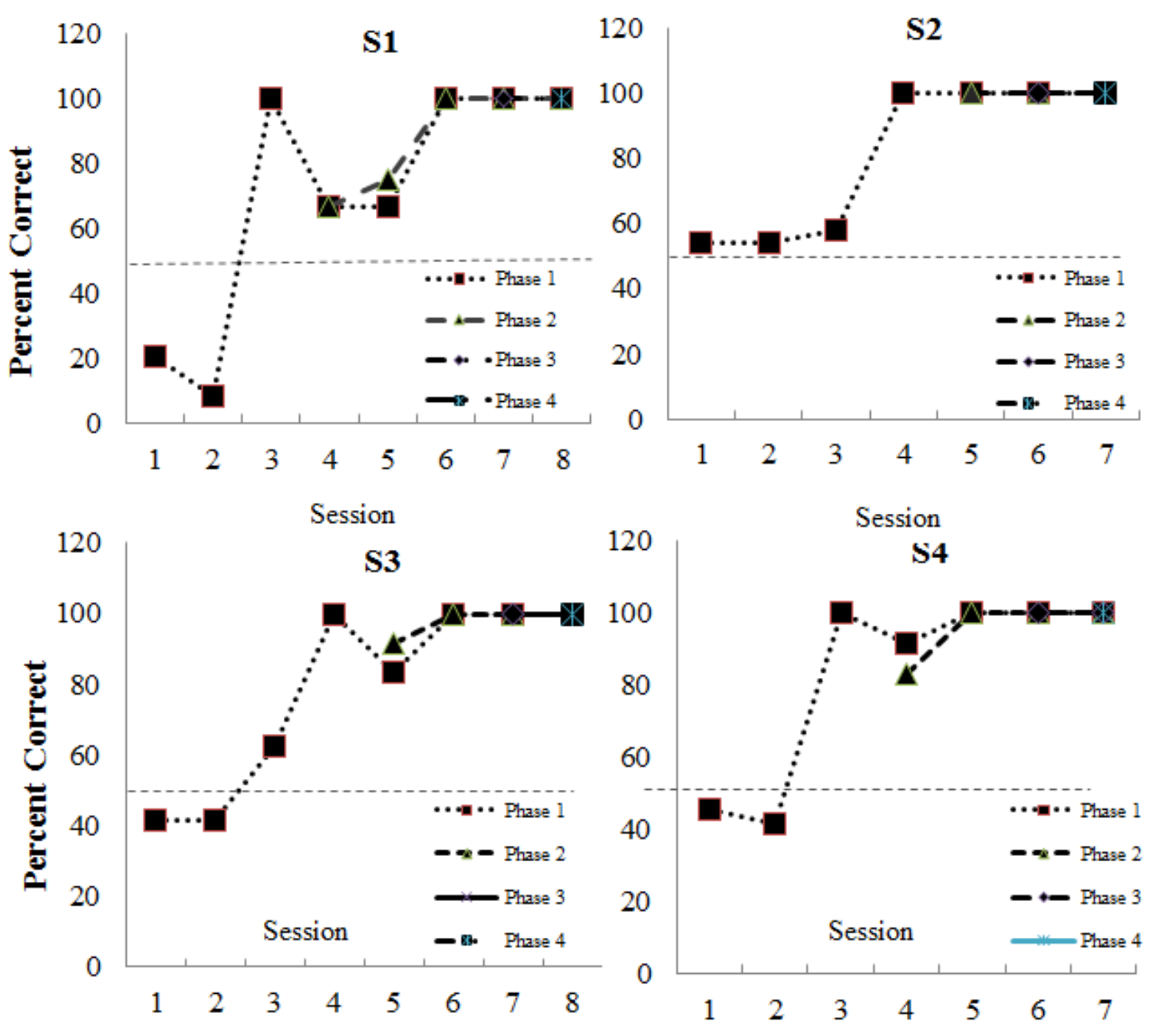

Figure 4. Acquisition training completed by S1,S2, S3 and S4. Dotted line represents the chance level performances 

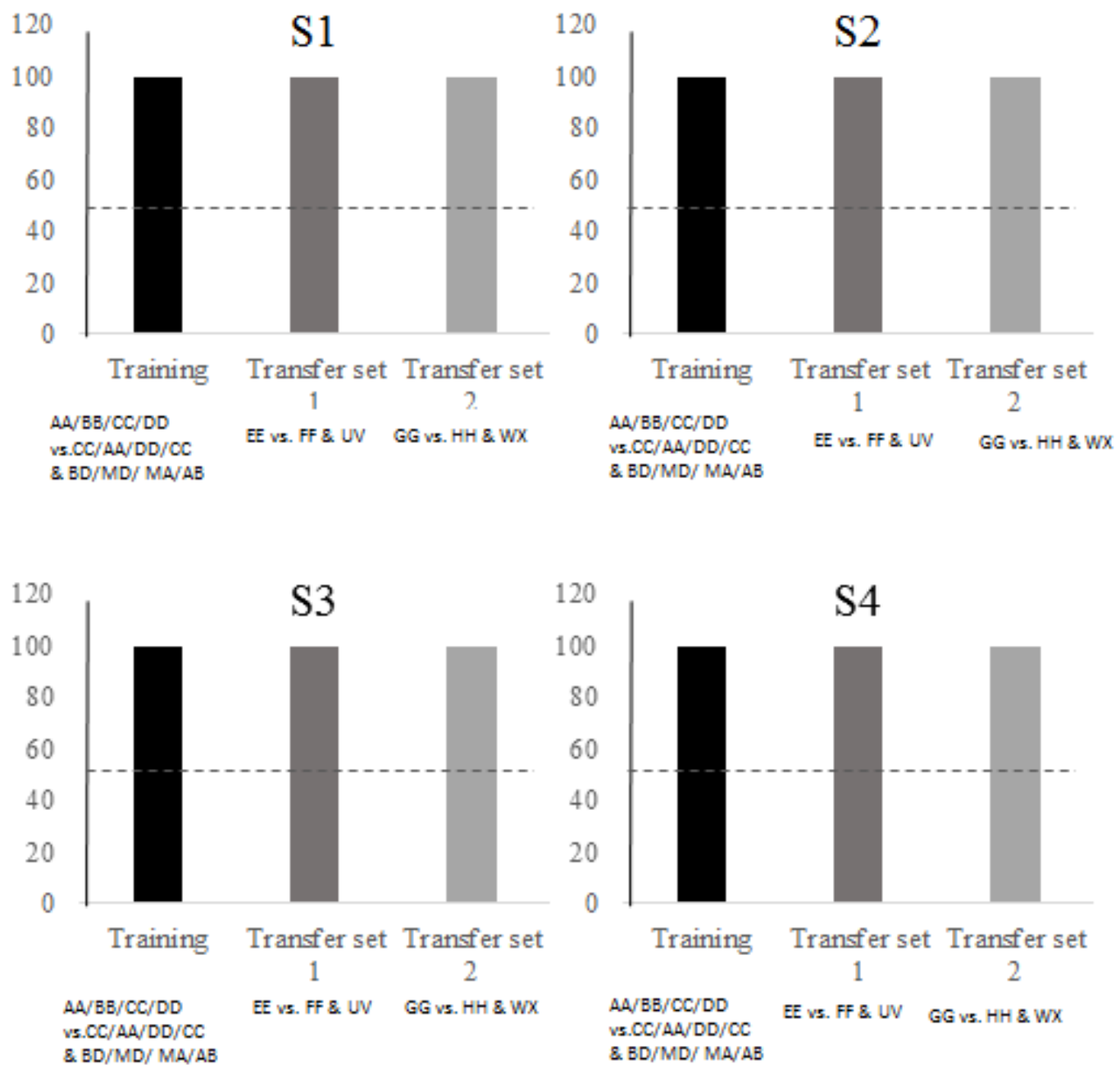

Figure 5. Training and transfer performances of S1, S2, S3, and S4. Dotted line represents the chance level performances

Some factors might contribute to concept formation in children. One of the major factors might be the concurrent training. As per concurrent training, a stimulus that is correct in a trial becomes incorrect in another trial. Such presentation of stimuli builds up an understanding in subjects that a particular stimulus or any stimulus does not lead them to the correct responses rather unearthing a relationship between or among the stimuli is the main way to solve the tasks thus propelling them to adopt a relational strategy to work out the discrimination tasks.

The present study used AA vs. BB \& MA as sample and comparison stimuli in phase 1. In this case, memorizing these three pair of stimuli (remembering the configurations of specific items called configural learning) was sufficient to make correct responses. In phase 2, AA and $\mathrm{BB}$ were concurrently used along with a different pair of stimuli MA that was used before. In this case too, configural learning (AA and BB and MA) might lead to the correct responses. But in phase 3 and in phase 4, a handsome number of pair of stimuli was generated from five training stimuli that produced a large number of configurations of stimuli that might make memorizing the number of tasks more difficult (Cook, Levison, Gillett, \& Blaisdell [30]) and might make the experimental subjects' memory load high. This difficulty might have forced the subjects to give up the memorizing strategy and switch to a more conceptual based strategy (an application of an abstract rule that can contribute to reducing the increased memory load (see also Santiago \& Wright [31]).

Here, it is worthy of mentioning that when many stimuli are presented, subjects have a tendency to remember some common properties of the stimuli by which they have the possibility to demonstrate some transfer of learning to the testing stimuli if the common properties of the training stimuli shares something common with that of the testing stimuli. If the subjects are able to constantly transfer the acquisition of training to the novel stimuli in the transfer test, such transfer of learning might be considered an abstract concept but with the possibility of restricted-domain relational learning ( learning limited to a specific area e.g., geometrical shapes). It is reported (Nakamura, Wright, Katz, Bodily \& Sturz [32]) that human learning might be relational but in a restricted domain that later in development gave way to 
relational factors (e.g., Chen \& Mo [33]).

Significant transfer may somewhat reflect generalization from training stimuli to transfer stimuli. Because larger number of stimuli may potentially increase the similarity between training and testing stimuli and such similarity may contribute to significant transfer. Therefore, it becomes difficult to rule out the possibility that transfer may be based on some common physical features shared between the novel stimuli and some of the stimuli used in training (Mackintosh [34]). Such a possibility might be plausible for the present study. Because a total number of 13 geometric and other shapes (five training stimuli and eight novel stimuli) were used in the present study. A good number of RMTS tasks were generated from these 13 shapes. In such stimuli setting, there might have an opportunity for the shapes to share some physical features between sample and comparison stimuli used in training and testing phases. However, to confirm to whether the findings of the present study are the products of stimulus generalization or restricted-domain relational learning, further study such as cross-modal study involving different mode of stimuli (e.g., a pair of two pens vs. a pair of cups and a different pair of stimuli namely a spoon and a pot) in which a pair of stimuli will not have an opportunity to share any perceptual features with other ones (training stimuli or stimuli used in intra-modal transfer test) might be considered (Tyrrell [35]).

Cross-modal transfer test has another pragmatic use. Subjects' abilities to respond to all potential dimensions of stimuli were not tested in the present study. Penn, Holyak and Povinelli [36] stated that to make an understanding about the abstract principle, one must learn to apply it to any stimulus dimension. Thus, as a part of future research, the present study should be extended to several cross-modal tests involving various novel sets of stimuli that transcend the boundary of stimulus dimension However, if human subjects cam exhibit robust transfer of learning to the cross-modal stimuli similar to that of intra-modal transfer, such cross-modal test performance is difficult to explain in terms of stimulus generalization from shape stimuli to cross-modal object stimuli because, generally speaking, it is hard to find any similar physical features between shapes and object stimuli .Conversely, if better performance in shapes-novel shapes transfer than in cross-modal transfer (e.g., shapes-objects) is demonstrated, it might be explained in terms of stimulus generalization. Interestingly, no individual difference was found in the relational performances of children except one who voluntarily left the experiment as he had the full independence to quit the experiment at any time (although his initial performances in phase 1 task praiseworthy [near $50 \%$ correct responses]) demonstrating that perceptual cues might not prevail in the experimental procedures. Thus, the children learned the tasks in similar manner, meaning that all four children were able to encode the relational properties from the stimuli in phase 1 and could apply this relational strategy to the tasks of other phases and finally to the novel pair of stimuli. If different learning strategy were in operation in them, the manner of their mastering the tasks would also be different. It is worthy of mentioning that in the case of S/D tasks, humans or animals have tendency to look for some specific features of stimuli and try to solve the tasks because it is the easiest way to pass the experimental situation. No deep thinking or complex brain functions are needed.

When they do not find out such possibility in stimuli set, they try to find out configural cues (configurations of specific items). In these two cases, different subjects might produce different types of performances. But when the subjects find no specific or configural cues from the stimuli set, they are bound to learn the concrete characteristics of the correct stimuli based on a consistent relationship between stimuli rather than perceptual stimulus characteristics. Notably, S/D tasks can not be solved by relying on the perceptual properties of a stimulus rather it should be worked out by learning concrete characteristics of stimuli in terms of its relationship with other ones. When there are possibilities of perceptual cues prevailing in the experimental tasks, the outcome of the experiment might not reflect on what the study actually likes to explore. However, the accuracy the children showed in the present study was comparable to or better than human studies with reinforcement training (e.g., Walker \& Gopnik [18]; Hochmann et al. [16]) suggesting that RMTS ability was the children's innate qualities (Anderson [37]) that did not seem to be affected by any external factors like reinforcement. Hence, it is clear that we, as the experimenter, did not facilitate them to acquire such higher-order cognitive ability rather they themselves mastered the RMTS tasks. We just provided them a suitable learning environment.

One possible explanation of the present study showing hefty acquisition and transfer of RMTS learning because the subjects used in the present study are older (7-8 years old) than other RMTS studies (e.g., Hochmann et al. [16] with 2-5 year olds; Christie \& Gentner [15] with 2-4 year olds; Walker \& Gopnik [18] with 18-30 month-olds; Walker, Bridgers \& Gopnik [17] with 18-48 month-olds). It is reported (cited in Premack [8]) that children below 4 years of old were not able to perform the XX-AA task and the XY-CD task. Children with ages of 4 and a half years old could perform the identical XX-AA task but they were still unable to perform the RMTS tasks indicating different relation (e.g., XY-CD). Children correctly performed both XX-AA and XY and CD tasks when they were 6 years old suggesting that age played a significant role in acquiring relational learning.

There is a physiological view of relational concept learning that explains (see a book authored by Carlson, N.R. [38]) that hippocampal lesion might show memory 
deficits particularly in the higher-order learning in animals and humans indicating that hippocampus areas, an important part of the limbic system, plays a vital role in the formation of higher-order cognitive functions like abstract concept learning. Older people have capacity to activate both left and right hippocampus, whereas younger people are able to activate only the left hippocampus. Furthermore, cognitive flexibility (the mental ability to switch between thinking about two different concepts and to think about multiple concept simultaneously), goal setting and information processing rapidly develop during 7-9 years old.

B.F. Skinner [39] studied animal (e.g., pigeons) behavior with reinforcement in an experimental setting and discovered learning ability in animals. With his operant conditioning theory, he stated that learning occurred in the presence of reinforcement. But the present study thinks that reinforcement might be mandatory for animals to acquire learning but this notion may not be true for humans in all cases. One explanation may be that although humans' brain shares something common with that of animals, the former one has some unique cognitive qualities as compared to animals. For example, humans have an ability to tackle the new situation with novel techniques. Due to having such creativity, humans can adapt with new situation and acquire new learning. Thus, reinforcement does not have to play a mandatory role in the acquisition of learning for humans but facilitates their learning in different aspects of their daily life. However, the present study with its remarkable findings without reinforcement training will further strengthen Bandura's social learning theory [40] stating that learning occurs through observation but without reinforcement and emerge as a challenge for the operant conditioning theory stating that the presence of reinforcement turns a trial in to learning.

Different species may process the RMTS tasks differently. Considering the phylogenetic and ecological disparities among humans and animal species (e.g., parrots, crows, and apes), there may be good reason to expect advanced conceptual abilities in humans if they are tested. Fagot, Wasserman and Young [41] observed in their comparative studies involving humans and baboons that humans correct responding to RMTS tasks was faster and higher than baboons. Furthermore, the conceptual processing of RMTS tasks was in more operation in humans than baboons. Despite having some similarity between the intelligence of humans and animals, the former ones have some superior cognitive skills that make them different from the latter ones. More specifically, humans have cognitive capacities to make combination of different types of information and knowledge with an aim to gain a new understanding about a particular subject and to apply the same rule or solution to one problem to a different and new situation (Hauser [42]). But such versatility in cognitive capacity does not seem to be available in animals.

Despite successful findings, some possible concerns originated from our study, that was, the pair of stimuli presented as sample one at every trial contained same relationship between itself. In RMTS tasks, subjects require choosing one of the two comparison stimuli based on the sample one. Therefore, subjects had to understand the same relationship in the entire experiment. Although it is acknowledged that subjects participating in the experiments had to understand the relationship among the pairs of stimuli (between pair of sample stimuli and one of the two pair of comparison stimuli resembling the sample one), one type of relational tasks (same relationship among pairs of stimuli) require same cognitive processing that might frame a mental set (responding to the stimuli based on same relationship) in the subjects thus making the discriminative tasks easy for them. Thus, they did not seem to pay a special effort to the understanding of the different pair of stimuli that was a part of the pair of comparison stimuli. If the subjects faced the relational tasks in terms of same and different relationship among sample and comparison stimuli, more difficult cognitive processing were required for solving the two-dimensional relational tasks thus demonstrating the cognitive flexibility of human brain functions.

The second possible candidate was that it employed just two items (MA) as different pair of stimuli in phase 1 and in phase 2 that made the discrimination tasks easier for the children to work out. Thus, if there were much variety in different pair of stimuli, these ones would be more competitive for the subjects that could propel them to use complex cognitive processing from the beginning of the experiment. In this case, reflection of more higher-order of cognitive ability would be visible.

The third one stated that we have employed five subjects in the present studies. In this case, more number of subjects could have been produced more reliable findings. Thus, future research should be conducted with larger number of subjects (at least more than 20 subjects).

\section{Conclusions}

To our knowledge, our findings are the first to show the RMTS abilities in children belonging to south Asian country particularly in Bangladesh. Although the findings of the present study were comparable or better than that of western cultures, the effects of age might be considered for the better performances in RMTS tasks showed by the children used in the present study. Earlier it was mentioned that a considerable number of attempts have been paid for the investigation of RMTS in humans some of which (e.g., Hochmann et al. [16]; Christie \& Gentner [15]) revealed the promising evidence in support of this higher-order cognitive abilities. Notwithstanding, there is still a remaining concern on what mechanisms are 
responsible for concept formation in humans or how it works in humans' brain is still unknown. The discovery of this mechanism may bring a breakthrough for the brain researchers by providing them an insight into the functions of a huge collection of neurons and synapses prevailing in the brain.

\section{Acknowledgements}

The authors profoundly appreciate all the people who have successfully contributed to ensuring this paper in place. Their contributions are acknowledged. However, their names can't be mentioned.

\section{REFERENCES}

[1] Katz, J.S., Wright, A.A., \& Bodily, K.D. Issues in the comparative cognition of abstract- concept learning.Comparative Cognition and behavior Review, Vol.2, 79-92, 2007.

[2] Wright, A. A. \& Lickteig, M.T. What is learned when concept learning fails?: A theory of restricted- domain relational learning. Learning and Motivation, Vol.41, 273-286, 2010.

[3] D' Amato, M.R., Salmon, D.P., \& Colombo, M. Extents and limits of the matching concept in monkeys (Cebus apella). Journal of Experimental Psychology: Animal Behavior Process, Vol.11, 35-51, 1985.

[4] Kastak, D. \& Schusterman, R.J. Animal Learning \& Behavior, Vol.22, 427-435, 1994. Online available from https://doi.org/10.3758/BF03209162.

[5] Boelens H1, Schenk J. Influence of identity versus Oddity pretraining on symmetric matching to sample. Psychological Reports, Vol.104, 861-873, 2009.

[6] Lazar, R. Extending sequence-class membership with matching to sample. Journal of The Experimental Analysis of Behavior, Vol.27, 381-392, 1977.

[7] Weinstein, B. Matching-from-sample by rhesus monkeys and by children. Journal of Comparative Psychology, Vol.31, 195-213, 1941.

[8] Premack, D. The codes of man and beasts. Behavioral and Brain Sciences, Vol.6, 125-167, 1983. Online available from http://dx.doi.org/10.1017/S0140525X00015077

[9] Smith, L. B. Young children's understanding of attributes and dimensions: A comparison of conceptual and linguistic measures. Child Development, Vol.55, 363-380, 1984.

[10] Fagot J, Thompson RKR Generalized relational matching by Guinea baboons (Papio papio) in two by two-item analogy problems. Psychological Science Vol.22:13041309,2011

[11] Wright, A. A. \& Katz, J. S. Mechanisms of same/different concept learning in primates and avians. Behavioral Processes, Vol.72, 234-254, 2006.
[12] Martinho, A.3rd, \& Kacelnik, A. Ducklings imprint on the relational concept of "same or Different". Science, Vol.353, 286-288, 2016. DOI: 10.1126/science.aaf4247.

[13] Thompson, R.K.1., Oden, D.L., \& Boysen, S.T Language-naive chimpanzees (Pan troglodytes) judge relations between relations in a conceptual matching-to-sample task. Journal of Experimental Psychology Animal Behavioral Process, Vol.23, 31-43, 1997.

[14] Obozova, T., Smirnova, A., Zorina, Z., \& Wasserman, E. Analogical reasoning in amazons. Animal Cognition. DOI 10.1007/s10071-015-0882-0, 2015.

[15] Christie, S. and Gentner, D. "Language Helps Children Succeed On A Classic Analogy Task". Cognitive Science, Vol.38, 383-397, 2014.

[16] Hochmann, J. R., Tuerk, A. S., Sanborn, S., Zhu, R., Long, R., Dempster, M., \& Carey, S. Children's representation of abstract relations in relational/array match-to-sample tasks, Cognitive Psychology, Vol.99, 17-43, 2017. https://doi.org/10.1016/j.cogpsych.2017.11.001.

[17] Walker, C. M., Bridgers, S., \& Gopnik, A. The early emergence and puzzling decline of relational reasoning: Effects of knowledge and search on inferring abstract concepts, Cognition, Vol.156, 30-40, 2016.

[18] Walker, C.M. \& Gopnik, A. Toddlers infer higher-order relational principles in causal learning. Psychological Science, Vol.25, 161-169, 2014.

[19] Chiao, J. Y., \& Blizinsky, K. D. Culture-Gene Coevolution of Individualism Collectivism and the Serotonin Transporter Gene. Proceedings of the Royal Society B, Vol.277, 529-537, 2010 https://doi.org/10.1098/rspb.2009.1650

[20] Chua, H. F., Boland, J. E., \& Nisbett, R. E. Proceedings of the National Academy of Sciences, Vol.102, 12629-12633, 2005. DOI: 10.1073/pnas.0506162102

[21] Masuda, T., \& Nisbett, R. E. Attending holistically versus analytically: Comparing the context sensitivity of Japanese and Americans. Journal of Personality and Social Psychology, Vol.81, 922-934, 2001. http://dx.doi.org/10.1037/0022-3514.81.5.922

[22] Masuda, T. \& Nisbett, R. E. Culture and change blindness. Cognitive Science, Vol.30, 381-396, 2006.

[23] Kuwabara, M., \& Smith, L. B. Cross-cultural differences in cognitive development: attention to relations and objects. Journal of experimental child psychology, Vol.113, 20-35, 2012.

[24] Smith, J., \& Tunick, J. Cross-modal transfer of a discrimination by retarded children. Journal of Experimental Child Psychology, Vol.7, 274-281, 1969.

[25] Birkimer, J. C. Control of responding by the elements of a compound discriminative stimulus and by the elements as individual discriminative stimuli. Journal of the Experimental Analysis of Behavior, Vol.12, 431-436, 1969.

[26] Daehler, M.W. \& Bukatko, D. Cognitive Development. Knopf, New York, 1985.

[27] Piaget, J. \& Inhelder, B. The Psychology of the child (H. 
Weaver, Trans.). NewYork: Basic Books, 1969. (Original work published 1966).

[28] James, W. The Principles of Psychology. New York: Dover Publications, Vol. 1. 1950. (Original work published 1890).

[29] Piaget, J. Origins of intelligence in the child. London: Routledge \& Kegan Paul, 1936.

[30] Cook, R. G., Levison, D.G., Gillett, S., \& Blaisdell, A.P. Capacity and limits of associative memory in pigeons. Psychonomic Bulletin \& Review, Vol.12, 350-358, 2005.

[31] Santiago, H.C. \& Wright, A.A. Pigeon memory: Same/different concept learning, serial probe recognition acquisition, and probe delay effects on the serial-position function. Journal of Experimental Psychology: Animal Behavior Processes, Vol.10, 498-512, 1984.

[32] Nakamura, T., Wright, A. A., Katz, J. S., Bodily, K.D., \& Sturz, B. R. Abstract concept learning carryover effects from the initial training set in pigeons (Columba livia). Journal of Comparative Psychology, Vol.123, No. 1, 79-89, 2009.

[33] Chen, Z. \& Mo, L. Schema induction in problem solving: A multidimensional analysis. Journal of Experimental Psychology: Learning, Memory, and Cognition, Vol.30, 583-600, 2004.

[34] Mackintosh, N.J. Abstraction and discrimination. In: Heyes, C., Huber, L. (Eds.), The Evolution of Cognition, MIT press, Cambridge, MA, pp. 123-141, 2000.

[35] Tyrrell, D.J. Crossmodal transfer of conceptual responding in children. Bulletin of the Psychonomic Society, Vol.4, 269-271, 1974.

[36] Penn, D.C., Holyoak, K. J., \& Povinelli, D.J. Darwin's mistake: Explaining the discontinuity between human and nonhuman minds. Behavior and Brain Sciences, Vol.31, 109-178, 2008.

[37] Anderson, J.R. Cognitive psychology and its implications (6th edn).New York: Worth Publishers, 2005.

[38] Carlson, N. R. Physiology of Behavior (6th Ed. P.465), 1998.

[39] Skinner, B.F. 'SUPERSTITION' IN THE PIGEON. Journal of Experimental Psychology, Vol.38, 168-172, 1948.

[40] Bandura, A. Social learning theory. Englewood Cliffs, N.J.: Prentice Hall, 1977.

[41] Fagot, J., Wasserman, E.A., \& Young, M.E. Discriminating the relation between relations: The role of entropy in abstract conceptualization by baboons (papio papio) and humans (Homo sapiens). Journal of Experimental Psychology: Animal Behavior Processes, Vol.27, 316-328, 2001.

[42] Hauser, M. Theory of "humaniqueness? Annual meeting of the American Association for the Advancement of Science. The Harvard Gazette. Feb. 14, 2008. 